\title{
Comparative Statistical Analysis of Pre-Existing Non-Symbolic Cognitive Math Models as a Predictor of Math Acuity in Children
}

\author{
Rohan Hundia
}

\begin{abstract}
Approximate Number System (ANS) acuity has been the underlying basis of mathematical magnitude measure in developing children. The early number learning in a child by means of numerosity representation is said to be best estimated by an ANS based model. ANS models are majorly specific to cases where numbers are represented by nondigits and are therefore non-symbolic (dot representations). Prior research also suggests that ANS acuity models could be used to give an estimate of an accuracy of a child in a non-symbolic math task. Common measures of ANS acuity are based on weber fraction based accuracy performance and some others are based on numerical distance effect and reaction time. However though, very few studies have amalgamated reaction time and weber fraction models and compared them at an individualistic level using actual data collected over participants.
\end{abstract}

In this research study, we effectively try to understand how factors like weber fraction, ratio, magnitude of numbers might affect the performance of a participant in a non-symbolic number comparison task. We also seek for any sort established relationship between numerical distance and reaction time and how that might be a predictor of good/bad performance. We carry out statistical analysis on both of these models (individually and combined) using data obtained from an online math task and thereby deduce which model could be a better predictor of a child's math acuity.

\section{PRIOR RESEARCH}

Research carried out in the past has majorly acknowledged that ANS is pivotal in the math learning of children and approximates numerical comparison on visual dot cues in both adults and children ${ }^{[1]}$ The research made use of two arrays of number dots and gathered data for human responses visual number comparison, addition and subtraction. Barth et al. concluded from their findings that the percentage of correct answers for visual comparison plummeted linearly as the ratio between the two number arrays increased. For a ratio (n1/n2) of 0.75 the accuracy was around $82 \%$ but then as the ratio increased to 0.86 the accuracy dropped to $67 \%$. They also deduced that the accuracy in addition tasks were substantially higher in addition tasks as compared to subtraction tasks and the reaction time of responses was higher for subtraction.

Similar research carried out by Halberda and Feigenson $(2008)^{[2]}$ came up with coherent results using weber fraction and psychophysical methods. They concluded that weber fraction could be a substantial indicator of math acuity, stating that Weber-Fechner have an inverse relationship with acuity and they decrease as age increases. For adults, the weber fraction value would be around 0.13 while as for 7 year olds it would be around 0.20 , so the weber value decreases as math learning acuity improves. The improvement of the 'number sense' still takes place during the ages of 7-10 and gets asymptotic during stages of adolescence.

Another research analysis by Park and Starns (2015) ${ }^{[3]}$ discussed a diffusion model approach considering both accuracy and RTs to approximate ANS and suggested how drift rate could be a better predictor of acuity than $w$ (weber value). They also generalised a relationship between RT and accuracy (> RT = higher accuracy value) as model performance and stated that $w$ and RT were highly correlated. This research was in contrast with the results found by Halberda et al. (2012) ${ }^{[4]}$ which concluded that there was no correlation between RT and weber values, only weber values and numerical distance were reasonable parameters to measure acuity in number comparison and basic arithmetic tasks.

\section{EXPERIMENTAL STUDY AND DATA COLLECTION}

Data of 198 participants was collected over an online math screener non-symbolic number comparison task whereby dot array images were numerically compared in a reaction time domain of $<2000$ milliseconds. The age range of the population was 7-18 with a mean age range of 11.81. The entire data set was stored in a csv file and the following metrics were stored in the file: $\mathrm{n} 1$ (numerical value of first image cue of dots), $\mathrm{n} 2$ (numerical value of second image cue of dots), ratio (n1/n2), session id and age of each subject, reaction time for each response and accuracy value for each question discretised as 1 or 0 for correct and incorrect answers respectively. The number of dot cues ranged from 1 to 96 for each image and the range of ratios was from 0.11 to 0.96 . The reaction time boundaries were from $450 \mathrm{~ms}$ to $2000 \mathrm{~ms}$ therefore any dataset out of these ranges was excluded during evaluation and termed as an outlier.

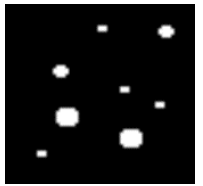

A

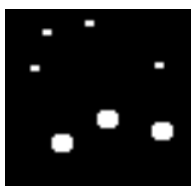

B

Fig 1. 1 A and B are non-symbolic representations in the form of dots for number comparison tasks in a 350 X 350-pixel block. (A) represents a cue of 8 dots 


\begin{tabular}{|c|c|c|c|c|c|c|}
\hline n1 & n2 & ratio & sid & RT & ACC & easyness \\
\hline & & & 382670 & & & \\
\hline 23 & 26 & 0.88 & 382670 & 478 & 1 & 0.761 \\
\hline 21 & 23 & 0.91 & 382670 & 618 & 1 & 0.691 \\
\hline 19 & 21 & 0.9 & 382670 & 526 & 1 & 0.737 \\
\hline 17 & 19 & 0.89 & 382670 & 490 & 1 & 0.755 \\
\hline 15 & 17 & 0.88 & 382670 & 668 & 1 & 0.666 \\
\hline 13 & 14 & 0.93 & 382670 & 505 & 1 & 0.7475 \\
\hline 11 & 12 & 0.92 & 382670 & 528 & 1 & 0.736 \\
\hline 9 & 10 & 0.9 & 382670 & 443 & 1 & 0.7785 \\
\hline 7 & 8 & 0.88 & 382670 & 463 & 1 & 0.7685 \\
\hline 5 & 7 & 0.71 & 382670 & 523 & 1 & 0.7385 \\
\hline 3 & 6 & 0.5 & 382670 & 428 & 1 & 0.786 \\
\hline 1 & 4 & 0.25 & 382670 & 485 & 1 & 0.7575 \\
\hline 4 & 6 & 0.67 & 382670 & 452 & 1 & 0.774 \\
\hline 3 & 11 & 0.27 & 382670 & 446 & 1 & 0.777 \\
\hline 2 & 6 & 0.33 & 382670 & 458 & 1 & 0.771 \\
\hline 1 & 4 & 0.25 & 382670 & 482 & 1 & 0.759 \\
\hline 3 & 9 & 0.33 & 382670 & 792 & 1 & 0.604 \\
\hline 25 & 28 & 0.89 & 382670 & 574 & 1 & 0.713 \\
\hline 27 & 33 & 0.82 & 382670 & 547 & 1 & 0.7265 \\
\hline
\end{tabular}

\begin{tabular}{|r|r|l|l|}
\hline sid & session & age & grade \\
\hline 869053 & 1 & & 6 \\
\hline 547182 & 1 & 11.43763 & \\
\hline 572942 & 2 & & 7 \\
\hline 270809 & 2 & & 7 \\
\hline 138354 & 1 & & 7 \\
\hline 356907 & 1 & & 7 \\
\hline 490573 & 1 & & \\
\hline 401743 & 1 & 5.582138 & 3 \\
\hline 572942 & 3 & & \\
\hline 982953 & 1 & & \\
\hline 875269 & 1 & & \\
\hline 972702 & 2 & & 7 \\
\hline 824438 & 1 & & 5 \\
\hline 636703 & 1 & 12.92749 & 5 \\
\hline 863772 & 2 & 10.43006 & 5 \\
\hline 283772 & 1 & 11.16377 & 5 \\
\hline 480101 & 1 & 10.77499 & 5 \\
\hline 773579 & 1 & 10.67095 & 5 \\
\hline 747661 & 1 & & 7 \\
\hline 972755 & 1 & 14.73429 & 9 \\
\hline 457804 & 1 & & 7 \\
\hline 785425 & 1 & 14.76725 & 7 \\
\hline
\end{tabular}

Fig 1.2 Depicts the data collected from an online math screener task and various parameters/features stored in two separate csv files for each specific subject. The sid (session id) was used to differentiate between subjects based on age and grade. The Easiness Value in the case was computed using the formula $\mathrm{E}=\mathrm{ACC}-(\mathrm{RT} / 2000.0)$

\section{DATA ANALYSIS}

\section{CORRELATION BETWEEN REACTION TIME AND ACCURACY:}

A few research studies in the past (Park and Starns - 2015) have shown that there is a linear coefficient based relationship between both reaction time - accuracy and reaction time - weber fraction. However, other research studies (Halberta et al. - 2012) argue that there is no correlation between reaction time and accuracy. In order to clear this ambiguity, statistical based analysis on reaction times and accuracy of 198 subjects was carried out.

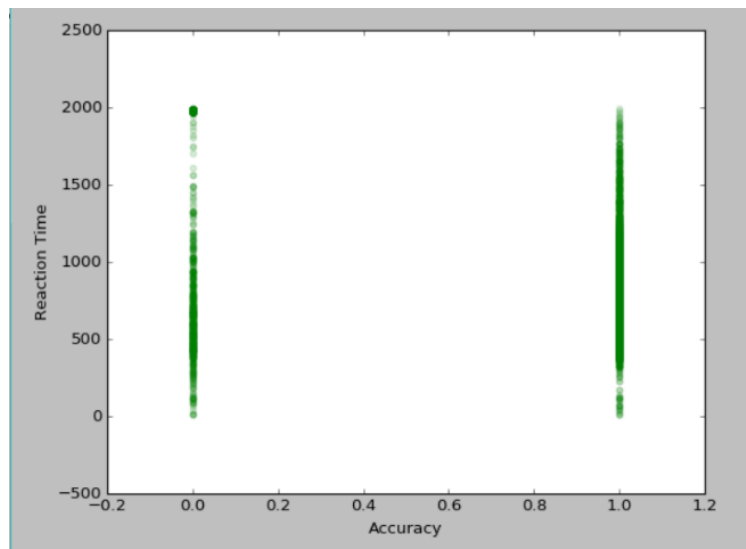

A

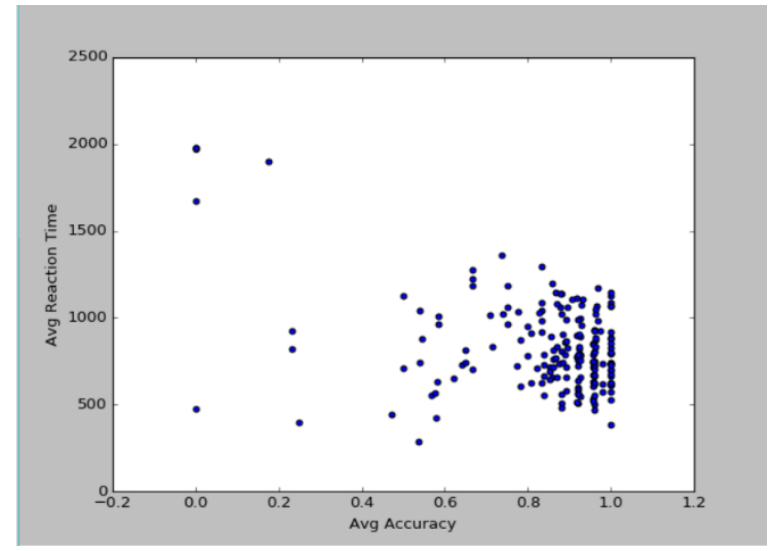

B

Fig 1.3 Scatterplots model RT vs Accuracy for all responses by subjects of all age groups. (A) shows accuracy as discrete values 0 or 1 for incorrect and correct respectively and depicts the reaction times for all responses. (B) depicts the average accuracy and average reaction time of a participant and plots for all participants.

No linear based correlation was deduced between RTs and Accuracy as depicted by Fig 1.3 A and B. However, it seemed clear from the data and plots (Fig $1.3 \mathrm{~A}$ ) that for higher RTs the number of incorrect responses was relatively low and vice versa. Therefore, it can be said that accuracy is relatively higher for higher reaction times. Fig $1.3 \mathrm{~B}$ on the other hand computes and plots average RTs and accuracy for each subject. The scatter plot depicts that for extreme values of RT $(>1500$ and $<500)$ there was low accuracy $(<0.5)$. Further analysis all showed that for subjects between age 8-12 the reaction time plot was more scattered as compared to teens or adults. This conclusively shows that the element of guessing is more common in 8-12 year olds. The number of correct answers for RT < 1000 for $12+$ year olds were almost 3 times higher as compared to $<12$ year olds which means that cognitive mathematical and "number sense" development still takes place till stages of pre-teen development.

\section{REACTION TIME, ACCURACY AND NUMERICAL DISTANCE EFFECT (NDE):}

One of the fundamental metrics in non-symbolic visual comparison of numbers is the numerical distance between the magnitude of the numbers represented. The NDE states that it is more difficult to differentiate numbers that are numerically close as compared to numbers which are numerically far apart. Research (Opstal and Verguts -2011$)^{[5]}$ suggests that the underlying cognitive process supporting NDE is the overlap of neural activation nodes leading to an overlap of tuning curves during number comparison tasks. Early research in 1967 (Moyer \& Landauer) ${ }^{[6]}$ described an inverse relationship between numerical distance and reaction time, however a few recent studies (Price et al. - 2012) ${ }^{[7]}$ haven't been able to replicate the same result and relationship using variant methods. Although the NDE approach has been quite prevalent in number response acuity and said to be correlated with both RTs and accuracy response, it is important to further investigate this approach. 
Method 1: Generating Scatter plot for RT and Numerical Distance and plot a line of best fit to get estimates of slope and RT intercept.

Method 2: Generating Scatter plot for Accuracy and Numerical distance and check for correlation.

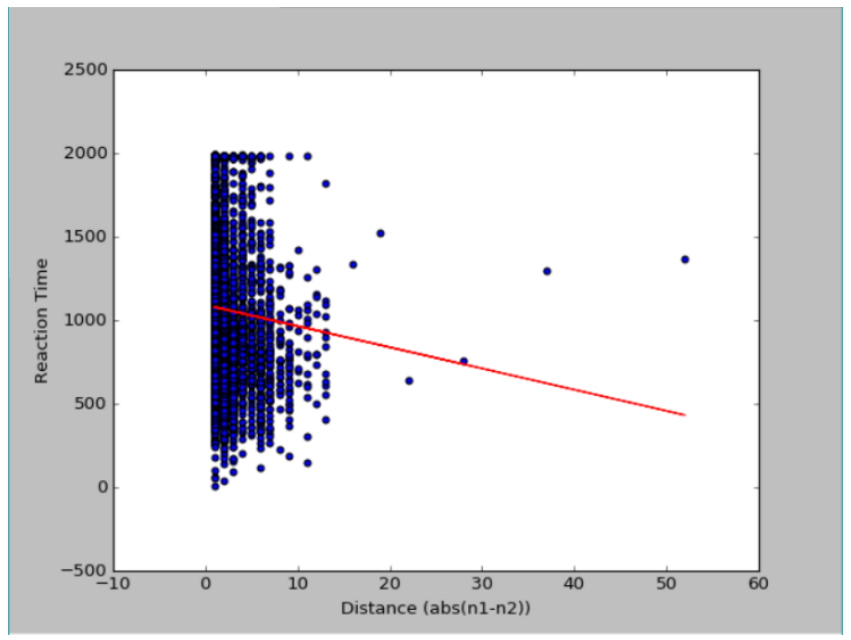

Fig 1.4 Scatterplot model RT vs ND with estimate line of best fit.

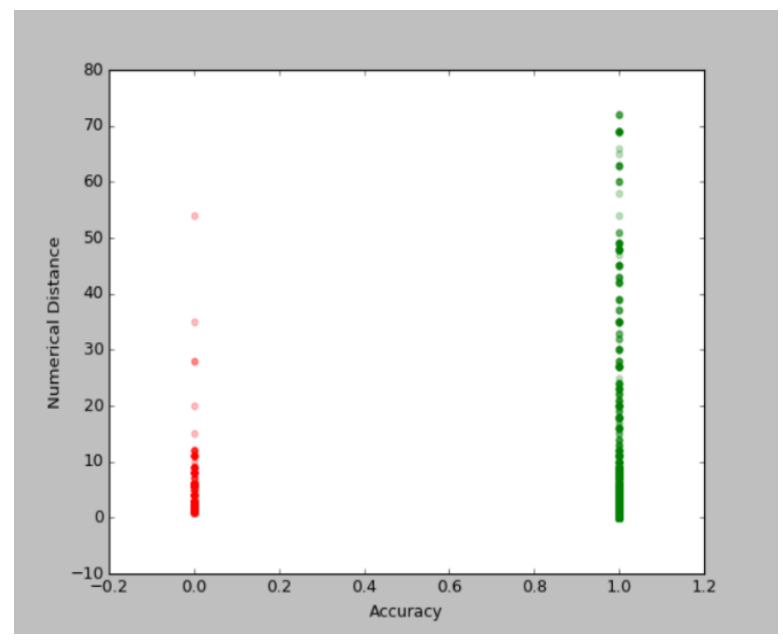

Fig 1.5 Scatterplot ND vs ACC (Red - Incorrect, Green - Correct Responses)

The line of best fit in Fig 1.4 does predict an inverse relationship between reaction time and numerical distance which is partially in congruity to prior research and correlation. The best fit has the following parameters $-\mathrm{m}=-12.91$ and $\mathrm{c}=1091.82$ where $\mathrm{m}$ and $\mathrm{c}$ are slope and intercept respectively. Although the best fit predicts inverse coefficient relation, it doesn't seem possible to model RTs solely based on numerical distance (since there is no modular parametric relationship).

It was also hypothesised that greater the numerical distance higher the probability of getting an answer correct. In order to justify the hypothesis a simple scatter plot comprising all NDs and Accuracy was graphed (Fig 1.5). The plot depicts that most of the incorrect responses was for ND < 10 and the percentage accuracy for ND > 20 was $94.23 \%$. This clearly verifies the numerical distance effect on accuracy, holding true for $>90 \%$ responses.

\section{EFFECT OF RATIO AND N1 ON INDIVIDUAL RTs:}

A statistical approach was used to model how Ratio and N1 could affect the response times of participants. To do the statistical simulation, a normal distribution was used to determine the RT for a given trial. For each numerical distance the standard deviation $(\sigma)$, variance $\left(\sigma^{2}\right)$ and mean of RTs were computed from the data set. Then the $(\sigma)$ values of RT averaged over all distances was computed. The averaged $\sigma$ (370 in this case) was used for drawing samples of RT from a normal distribution curve. The obtained samples of RT were plotted as a colormap on N1 vs Ratio Curve.

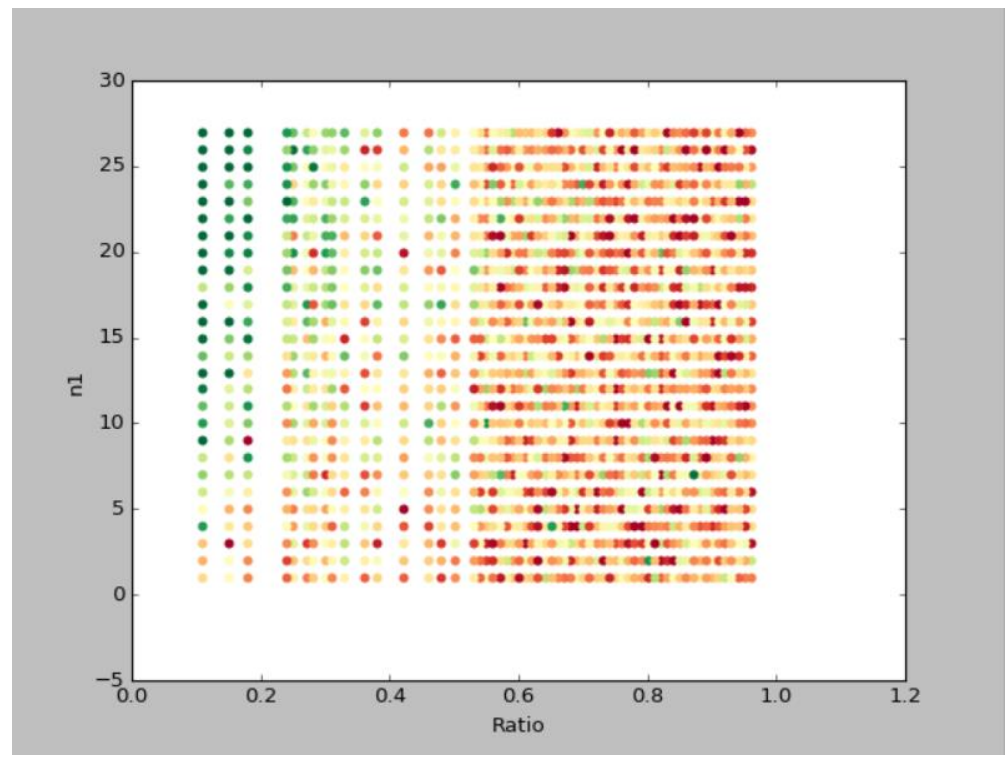

Fig 1.6 Scatter Plot of N1 and Ratios with the colour map (Green $\rightarrow$ Red) showing an increase in RT. RT values were sampled from a normal distribution with average $\sigma=370$. The RTs transition at approximately a ratio value of 
The RT colormap (Fig 1.6) conclusively depicts that as the ratio increases the response reaction time increases. Also, the $\mathrm{n} 1$ values decrease RT increases. The line demarcating a transition from low RTs to higher RTs could be said to be diagonal from the graph plotted above. The plot above could be supported by intuitive understanding of how math learning takes place in children. As the ratios between N1 and N2 increase (irrespective of the Numerical Distance), it becomes difficult to differentiate between non-symbolic number arrays and hence there is an increase in response time. For smaller N1s the numerical distance (given a fixed ratio) is lower as compared to higher N1s (with that same ratio). Therefore, the results follow the NDE by depicting that higher response time correlates to lower numerical distance.

\section{WEBER FRACTION AND THE APPROXIMATE NUMBER SYSTEM (ANS):}

The major underlying basis of the ANS has been the Weber fraction whereby the ability to discriminate between two non-symbolic quantities is most dependent on the ratio of the two quantities. Prior research (Feigenson et al. -2004$)^{[8]}$ states that the approximated number system always follows the Weber's Law and therefore the ability to discriminate between quantities does not depend on the magnitude of number or the numerical distance. With the result analysis carried out above, it can be stated that the Weber's Law doesn't completely hold true as its depicted above how the accuracy also depends on the numerical distance. This section would explore the weber fraction model and its predictive analysis of accuracy and easiness independent of the NDE.

The Probability of Accuracy (P_ACC) can be modelled using weber fraction by the formulas given below:

P_ERROR under a normal curve $=\frac{1}{2} \operatorname{erfc} \frac{(|n 1-n 2|)}{\sqrt{2} w \sqrt{n 1^{2}+n 2^{2}}} \quad$ where $\mathrm{w}=$ weber fraction value

P_ACC $=1-$ P_ERROR

The weber fraction value is the difference between the larger number and the smaller number divided by the smaller number.

Random sampling of the accuracy values is done on the basis of the obtained P_ACC value for each combination of $\mathrm{N} 1$ and $\mathrm{N} 2$ and the weber fraction value (based on age).

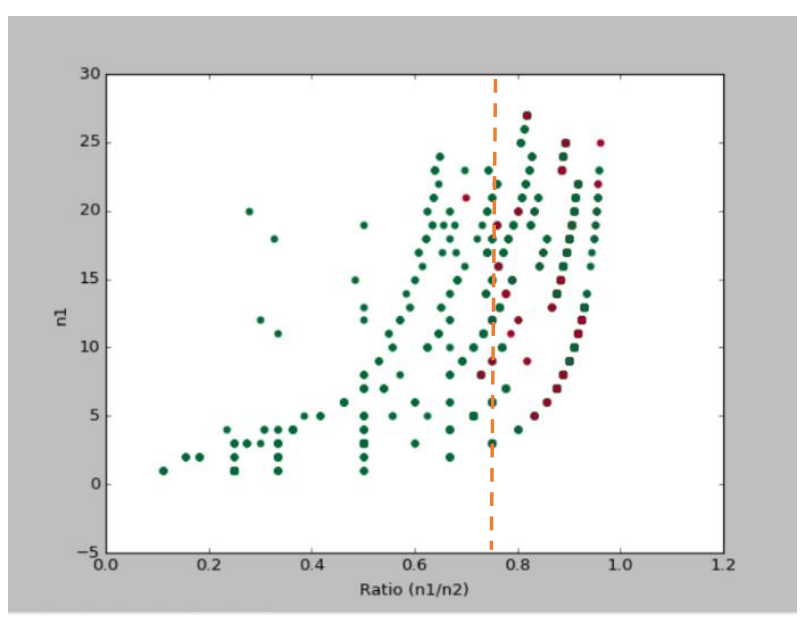

A

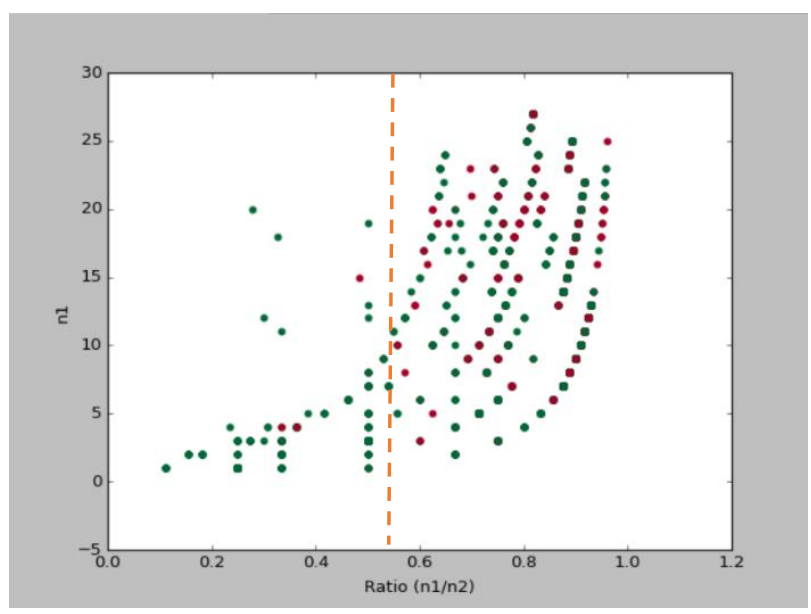

B

Fig 1.7 Scatter Plot of N1 and Ratios with the colour map based on discrete accuracy. Green - Correct Responses (Acc = 1) and Red Incorrect Responses (Acc $=0$ ) whereby accuracy is sampled from P_ACC and Error formula. For (A) weber fraction value is 0.15 and for (B) weber fraction value is 0.35

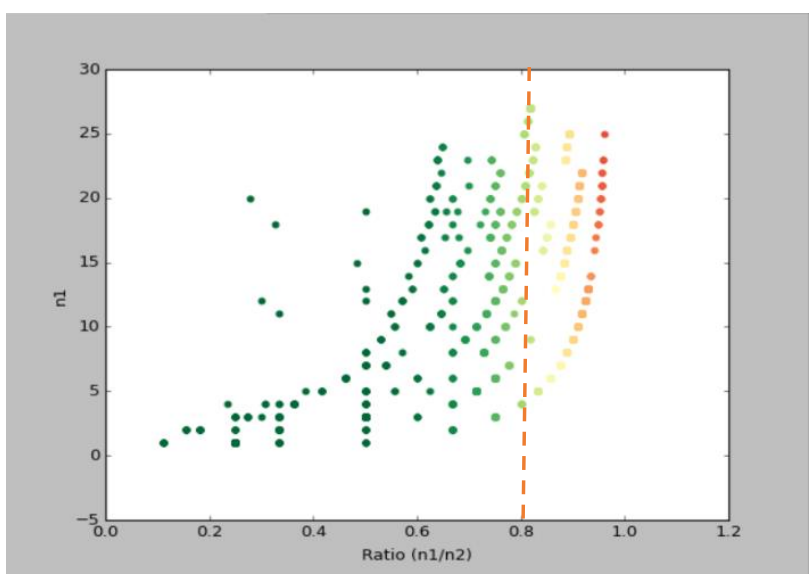

A

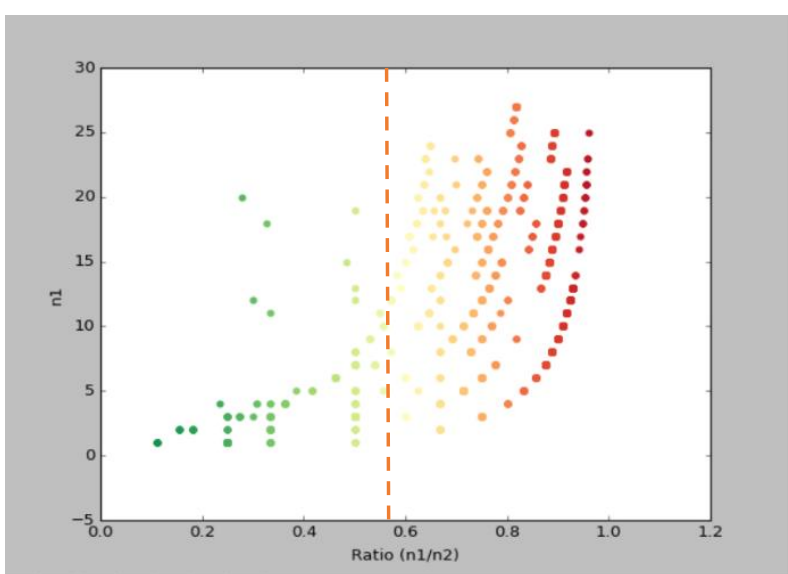

B

Fig 1.8 Scatter Plot of N1 and Ratios with the colour map based on P_ACC. Shift from Green to Red depicts a decrease in level of P_acc. For $(\mathrm{A})$ weber fraction value $=0.15$ and for $(\mathrm{B})$ weber fraction value $(\mathrm{B})$ weber fraction value is 0.35 
The accuracy on a trial decreases as the ratio increases which is supported by the graphs above. Fig 1.7 (A) depicts a graph with weber fraction value 0.15 (in Adults) and shows that the simulated incorrect responses occur when ratio is $>0.77$ (as depicted by the orange line). Fig 1.8 (A) also supports the previous inference by showing that the $\mathrm{P}_{-}$Acc values start to decrease below 0.5 when ratio is higher than 0.8 .

Similarly, Fig 1.7 (B) uses a weber fraction of 0.35 (in 4 year olds) to generate $\mathrm{P}$ _Acc values and sample Acc values ad shows that most incorrect responses occur when ratio $>0.56$ (orange line in B). Supporting the previous claim, Fig 1.8 (B) shows similar results and color map.

The number of incorrect responses in (A) are also significantly lower than in (B)

The vertical shift and change in number of incorrect responses in the graphs above is due to an increase in weber value which explains that how the weber value decreases during stage of math acuity development and has a strong correlation with performance in a non-symbolic number comparison task.

\section{SIMULATED EASINESS VALUES:}

Statistical analysis was carried out to understand how 'simulated easiness' on a task based on Weber Fraction and RT correlated with ratio and N1. Various simulated person instances were created based on weber values, $\mathrm{m}$ and intercept as depicted by the table below:

\begin{tabular}{|l|r|r|r|}
\hline Person Instances & Weber Fraction Value Slope (Based on RT) & Intercept (Based on RT) \\
\hline Person 1 & & & \\
\hline Person 2 & 0.29 & -12.9 & 900 \\
\hline Person 3 & 0.29 & -12.9 & 1400 \\
\hline Person 4 & 0.29 & -50 & 1400 \\
\hline Person 5 & 0.14 & -12.9 & 900 \\
\hline Person 6 & 0.14 & -12.9 & 1400 \\
\hline
\end{tabular}

The underlying purpose of the statistical simulation was to understand how the Easiness on a task was dependent on Weber Fraction, Slope and Intercept. The formula below was used to compute Easiness for a combination of N1 and Ratio:

Easiness $=$ Accuracy - RT/2000.0

where accuracy was obtained from sampling over $P_{-}$Acc values and RT was sampled from a normal distribution as above

Scatterplot colour map as below were produced (taking into account 10\% of response guessing in each case):

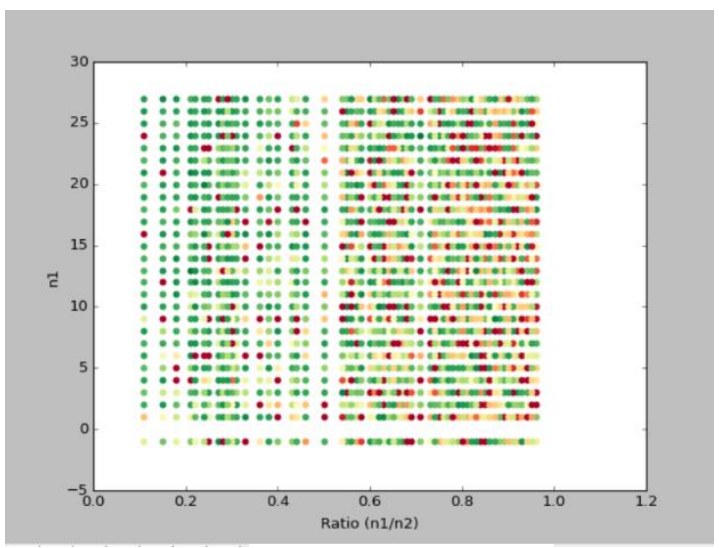

Person 1

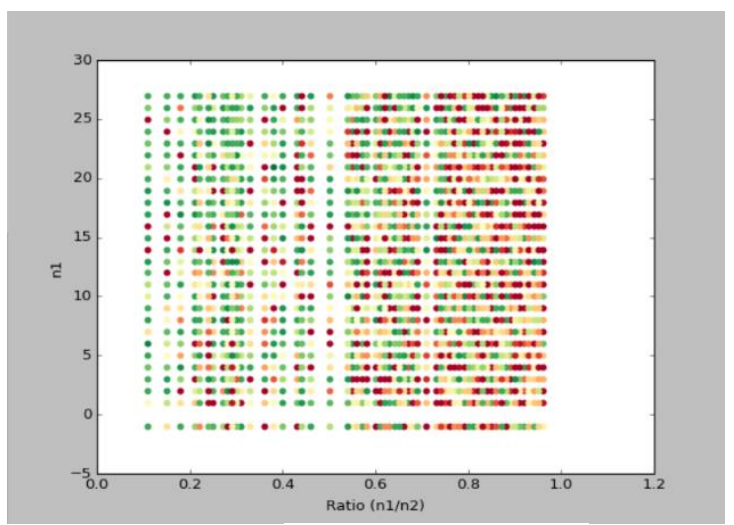

Person 2

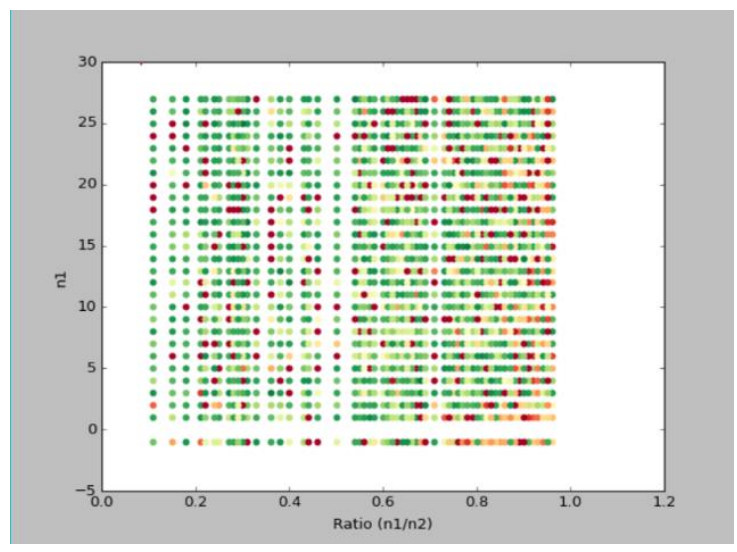

Person 4

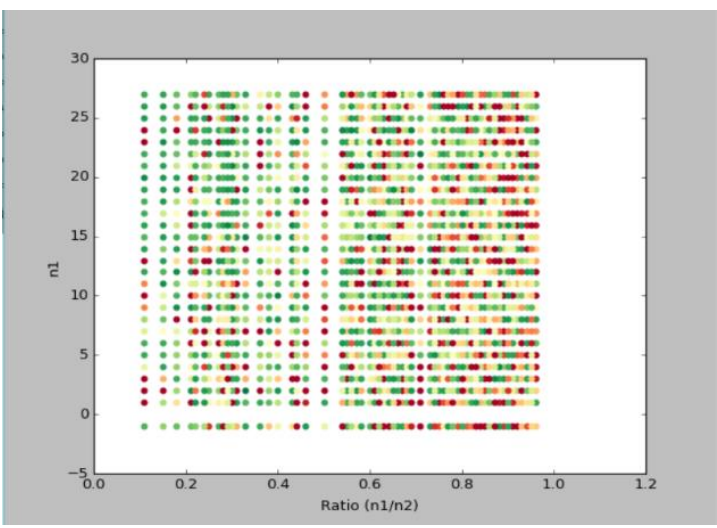

Person 5 


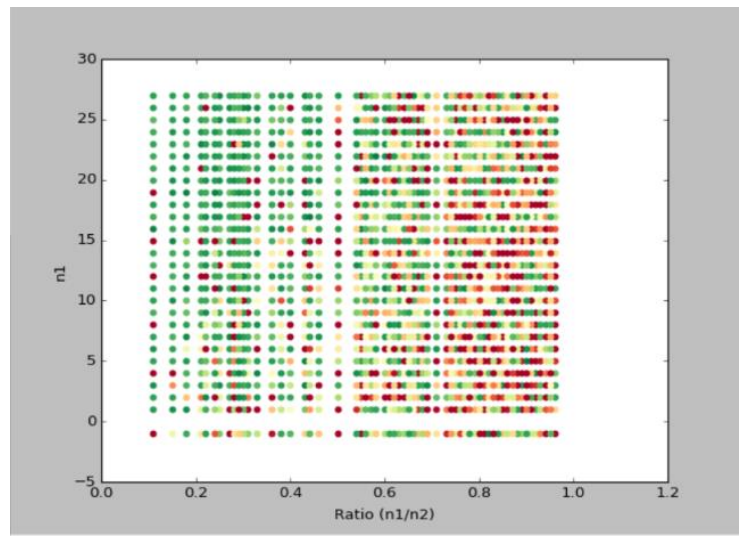

Person 3

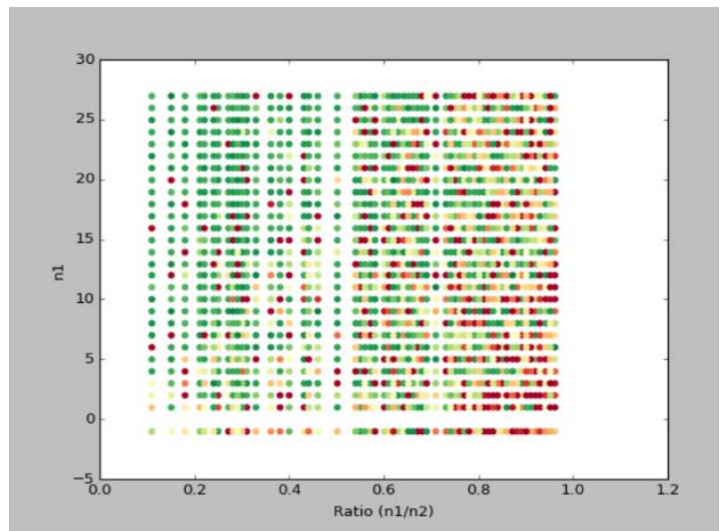

Person 6

Fig 1.9 Scatter Plots of N1 and Ratios with the colour map based on simulated P_Acc values (with 10\% random guessing) for various values of Weber Fraction, Slope and Intercept based on Person Instances in the table above.

The following deductions can be made from the plots above:

- $\quad$ Lower Weber Fraction = Higher Non-Symbolic Math Acuity

This claim is supported by comparing Fig 1.8 (Person 1 and 4), (Person 2 and 5), (Person 3 and 6). In each of these comparisons, the only parameter that changes is the weber fraction value. For example, the P_Acc values in Person 4 is higher than in Person 1 as shown by scatter plot, since the density of red and yellow error dots is higher in Person 1.

- $\quad$ Higher Intercept $=$ Lower Acuity

This claim is supported by comparing Fig 1.8 (Person 1 and 2), (Person 4 and 5). In these comparisons values of weber fraction and slope are kept constant and only the intercept changes. Plot comparison of Person 1 and 2 clearly shows the density of errors is higher in Person 2 compared to Person 1 which is caused due to Person 2 having a higher RT intercept.

- Slope obtained from RT vs ND graph has no substantial effect on math acuity for closer values. However, more negative the slope higher the acuity.

\section{COMPARATIVE STATISTICAL ANALYSIS BETWEEN ACTUAL ACCURACY AND SIMULATED} ACCURACY VALUES:

A final analysis approach was used to conclude how efficiently could the weber fraction be used to predict accuracy on task. Therefore, a model was created whereby the participants were classified into various weber fraction, slope and intercept values based on their age and grade. The table below represents the following classification:

\begin{tabular}{|l|r|r|r|}
\hline Age & Weber Fraction Value Slope (Based on RT) & Intercept (Based on RT) \\
\hline$<=7$ & 0.22 & -15 & 1000 \\
\hline 8 to 10 & 0.19 & -25 & 800 \\
\hline 11 to 14 & 0.16 & -35 & 700 \\
\hline 15 to 18 & 0.13 & -45 & 500 \\
\hline
\end{tabular}

Method: Simulated Easiness values were produced using P_ACC formula and sampled RT with the weber fraction, $\mathrm{m}$ and intercept values above specific to age for each combination of $\mathrm{N} 1$ and Ratio. The simulated Easiness values were then compared to the actual easiness values stored in the csv file (at the time of task performance). The same was done for the simulated RTs and actual RTs. The graph results were as follows:
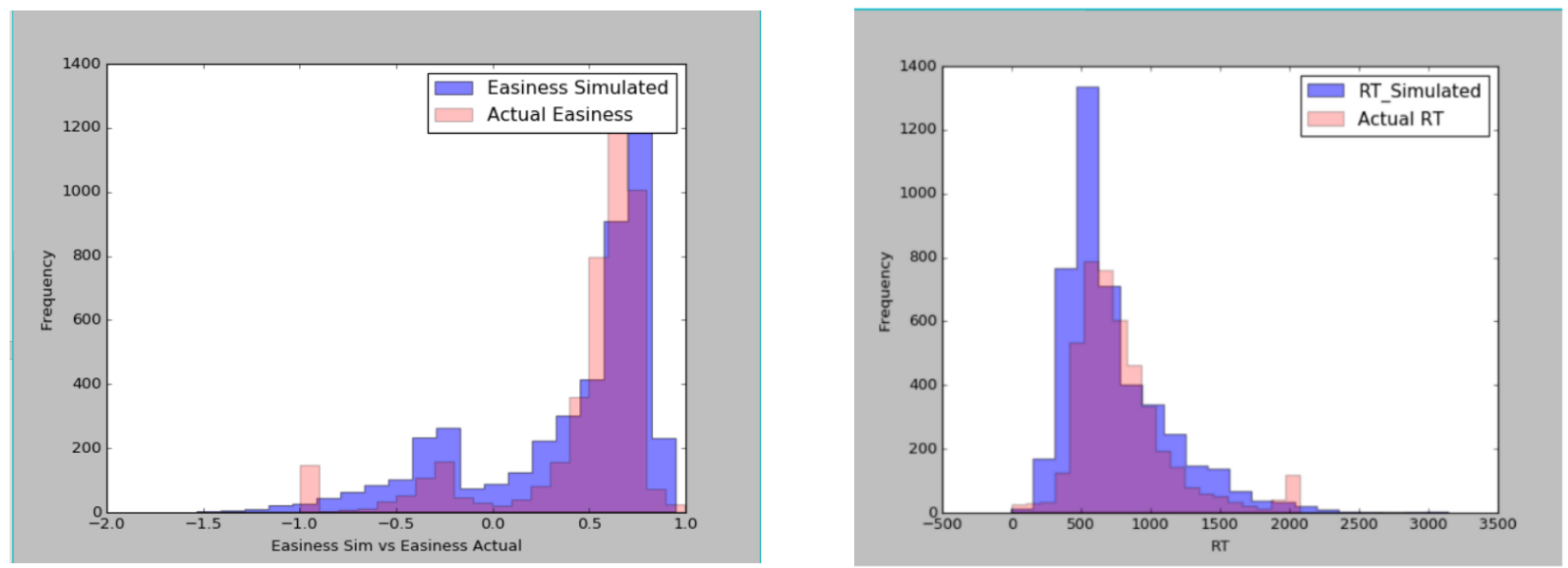

Fig 2.0 Histogram Plot for both simulated RT vs Actual RT and Simulated Easiness vs Actual Easiness 
Both the histogram plots above show high overlap between actual and simulated values which confirms that the weber fraction simulation model works with high accuracy in predicting actual data (both Easiness and RT). However, in both the plots the actual plot overestimates the simulated plot and the cause for that could probably be the NDE driving the RT which is independent of the weber model.

Comparison was also carried out between simulated discrete accuracy values and actual accuracy values. The simulated values matched the actual values in $\mathbf{7 2 . 3 \%}$ cases which means that the weber fraction model works with 72.3 correctness in predicting whether a participant would get an answer right or wrong.

\section{CONCLUSION AND FUTURE WORK}

The findings highlight several properties on which the performance of a participant depends in a number comparison task. The two major factors being weber fraction (dependent on the ratio) and the numerical distance effect. However, the RT/ND model could not model the accuracy of a participant in a trial. While the weber fraction-based model worked with $72.3 \%$ correctness in predicting the accuracy performance in a trial. It should be noted however that the weber model overestimated the Easiness of a trial in majority of cases. Therefore, further analysis is required to determine whether the overestimation was caused due to the NDE driving the RT or random cognitive based guessing $(>10 \%)$ by participants in trials. The findings also highlighted various correlations between cognitive based parameters on which the accuracy of a participant trial may depend. It was also found that the amalgamation of the NDE and weber model was less accurate than the weber model itself, since there was no perfect model to predict RT based on NDE (due to ambiguous correlation).

Future work would include investigations on how the NDE model actually drives the RT and therefore one of the possibilities could be to use a Metropolis Hastings algorithm to look for any probabilistic based approach to parametrize ND and RT and look for correlations. Creating an algorithmic equation which can generate RT as a function of ND could be super helpful. After this, further statistical tests could be used to explore how RT/ND models could be clubbed together with Weber fraction models.

\section{REFERENCES}

1. Barth H., LaMont K., Lipton J., Dehaene S., Kanwisher N., Spelke E. S. (2006). Non-symbolic arithmetic in adults and young children. Cognition 98

2. Halberda J., Feigenson L. (2008). Developmental Change in the Acuity of the "Number Sense": The Approximate Number System in 3-, 4-, 5-, and 6-Year-Olds and Adults. Developmental Psychology Vol.4

3. Park J., Starns J. (2015). The Approximate Number System Acuity Redefined: A Diffusion Model Approach. Frontiers in Psychology.

4. Halberda J., Ly R., Wilmer J. B., Naiman D. Q., Germine L. (2012). Number sense across the lifespan as revealed by a massive Internet-based sample.

5. Opstal F., Verguts T. (2011). The origin of numerical distance effect: The same-different task. Journal of Cognitive Psychology.

6. Moyer, R. S., \& Landauer, T. K. (1967). Time required for judgments of numerical inequality. Nature.

7. Price, G. R., Palmer, D., Battista, C., \& Ansari, D. (2012). Nonsymbolic numerical magnitude comparison: Reliability and validity of different task variants and outcome measures, and their relationship to arithmetic achievement in adults. Acta psychologica

8. Feigenson L., Dehaene S., Spelke E. (2004). Core systems of number. Trends Cognitive Science 